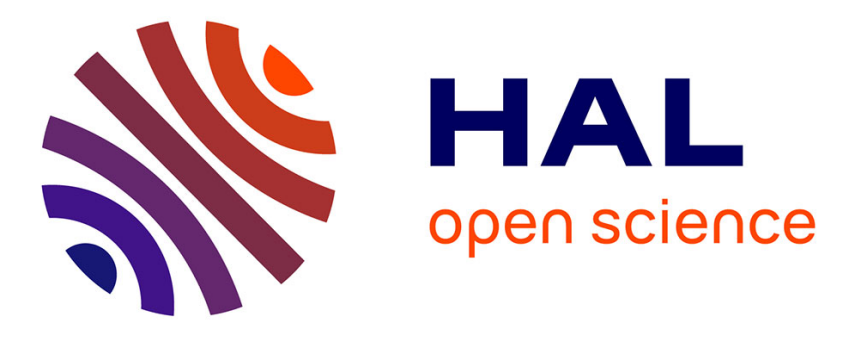

\title{
Assessment of on-body skin-confined propagation for Body Area Network,
}

Qiang Zhang, Julien Sarrazin, Massimiliano Casaletti, Philippe de Doncker, Aziz Benlarbi-Delai

\section{- To cite this version:}

Qiang Zhang, Julien Sarrazin, Massimiliano Casaletti, Philippe de Doncker, Aziz Benlarbi-Delai. Assessment of on-body skin-confined propagation for Body Area Network,. IEEE Antennas and Wireless Propagation Letters, 2017, 16, pp.2610 - 2613. 10.1109/LAWP.2017.2735631 . hal-01581037

\section{HAL Id: hal-01581037 https: / hal.sorbonne-universite.fr/hal-01581037}

Submitted on 3 Sep 2019

HAL is a multi-disciplinary open access archive for the deposit and dissemination of scientific research documents, whether they are published or not. The documents may come from teaching and research institutions in France or abroad, or from public or private research centers.
L'archive ouverte pluridisciplinaire HAL, est destinée au dépôt et à la diffusion de documents scientifiques de niveau recherche, publiés ou non, émanant des établissements d'enseignement et de recherche français ou étrangers, des laboratoires publics ou privés. 


\title{
Assessment of on-body skin-confined propagation for Body Area Network
}

\author{
Qiang Zhang, Julien Sarrazin, Member, IEEE, Massimiliano Casaletti, Member, IEEE, Philippe De
}

Doncker, and Aziz Benlarbi-Delaï

\begin{abstract}
This letter assesses the potential of using surface waves to implement secure on-body communications. The propagation medium being the human skin, a transverse resonance method is used to analyze existing modes within the multilayered structure that composes the human tissues. Confinement capabilities as well as propagation losses are investigated for frequency up to $60 \mathrm{GHz}$. It is shown in particular that the 3-10 GHz band could allow secure communications up to few tens of centimeters. This frequency band is furthermore considered as Ultra Wideband channels in the IEEE 802.15.6 standard dedicated to Body Area Networks.
\end{abstract}

Index Terms-Body Area Networks (BAN), propagation, multilayered human body model, dispersion analysis, on-body communication, transverse resonance method

\section{INTRODUCTION}

I $\mathrm{n}$ the past decade, wireless communication systems around the human body have attracted an increasing attention from researchers. Due to aging population issues, the intensive care units in hospital have an arising need of real-time remote monitoring and treating for patients. This task may be achieved by Body Area Networks (BANs), which interconnect wearable sensors and actuators around human body with an external interface. IEEE 802.15.6 standard has been established in order to provide a short-range, extremely low power, highly reliable wireless communications in BANs with data rates up to 10 Mbps [1].

Since the transmitted information is user's private vital signs, such as heart rate and blood pressure, high data security is required in the system. However, the wireless communications have an unbounded nature, which makes the BAN vulnerable to interception. A robust approach to enhance the security is to ensure it at the physical layer. Three physical layers are considered in the IEEE 802.15.6 standard: ultra-wideband

This paragraph of the first footnote will contain the date on which you submitted your paper for review. This work was performed within the Labex SMART supported by French state funds managed by the ANR within the Investissements d'Avenir programme under reference ANR-11-IDEX-0004-02

Q. Zhang, J. Sarrazin, M. Casaletti, and A. Benlarbi-Delai, are with Sorbonne Universités, UPMC Univ Paris 06, UR2, L2E, F-75005, Paris, France (e-mail: $\quad$ qqiang.zhang, julien.sarrazin, massimiliano.casaletti, aziz.benlarbi_delai\}@umpc.fr).

L. Petrillo and P. De Doncker are with OPERA Dpt. - Wireless Communications Group, Université Libre de Bruxelles (ULB), B-1050 Brussels, Belgium (e-mail: \{lpetrill, pdedonck\}@ulb.ac.be)
(UWB) PHY [2], narrowband (NB) PHY [3], and human body communication (HBC) PHY [4]. HBC uses the human body as a transmission medium, which inherently confines the signal to the body area thereby exhibiting interesting features regarding privacy issues. At $16 \mathrm{MHz}$ and $27 \mathrm{MHz}$ (i.e. operating frequencies considered for $\mathrm{HBC}$ in the standard), capacitive coupling exhibit much higher power efficiencies than galvanic coupling [4]. However, with capacitive coupling, the signal is less confined within the body and performance is strongly environment-dependent. Indeed, the capacitance between the floating electrode of sensors and the environment directly influences the received power, thereby making a robust implementation tricky.

While NB and UWB on-body propagation at few hundreds of megahertz up to a few gigahertz have been widely investigated [5]-[9], the confinement of on-body surface waves (SW) inside the human skin as a solution for secure communications has not been paid much interest. Authors in [5] investigated the wave propagation using a multilayered human model. However, a single geometry has been studied and few physical insights have been given. Consequently, the goal of this letter is to study the propagation mechanisms that take place within the skin in order to assess their suitability to support a secure communication between on-body sensors. To that aim, section II introduces the topology of the multilayer geometry that is used to model the human tissues and results including confinement capabilities and propagation losses are presented in section III. Conclusion and perspective are given in section IV.

\section{Multilayered Human Body Model}

On-body propagation is often studied using full-wave simulations with complete human body models. However, in addition to be a large numerical problem when frequency gets higher, it does not enable a full understanding of propagation phenomena. Consequently, a simpler model modeling the human tissues as a flat multilayer geometry is considered such as in [5], which enables determining the discrete propagation modes that can be supported by such a structure. Flat geometries have been shown to be valid enough in the case of on-body communications using Norton theory for instance [10].

As presented in Fig. 1, each layer represents a different human tissue (i.e. skin, fat, and muscle) characterized by a complex permittivity $\varepsilon=\varepsilon_{0} \varepsilon_{r}=\varepsilon_{0}\left(\varepsilon_{r}^{\prime}-j \varepsilon_{r}^{\prime \prime}\right)$, where $\varepsilon_{0}$ is 
the vacuum permittivity, $\varepsilon_{r}$ is the body tissue relative permittivity, and an $e^{j \omega t}$ time convention is considered. The permittivity values for skin, fat, and muscle can be found in [11] for frequencies ranging from $10 \mathrm{~Hz}$ up to $100 \mathrm{GHz}$. According to different locations and different persons, skin thickness was found in medical documents ranging from 0.1 to $5 \mathrm{~mm}$, and fat from 1 to $30 \mathrm{~mm}[12]-[15]$. In most parts of human body, such as forearm and chest, skin thickness is about $1 \mathrm{~mm}$. In this paper, various skin and fat thicknesses are considered in order to analyse how these properties influence the on-body propagation.

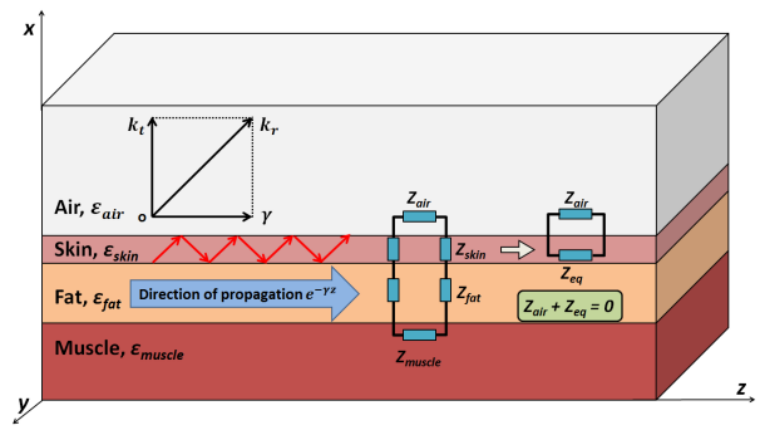

Fig. 1. Multilayered human body modeling and Transverse Resonance Method mechanism

The 4-layers air-skin-fat-muscle model is presented in Fig. 1. Dimensions along $y$-axis and $z$-axis (direction of propagation) are supposed infinite. In addition, the air and muscle layers are also supposed infinite in the positive and negative $x$-axis direction respectively. The complex intrinsic wavenumber of each layer $k_{i}$, at a given frequency, is determined as $k_{i}=$ $\omega \sqrt{\varepsilon_{i}(\omega) \mu_{0}}$, where $\omega$ and $\mu_{0}$ represent the angular frequency and the vacuum permeability respectively, and $\varepsilon_{i}(\omega)$ is the frequency-dependent complex permittivity of the tissue " $i$ ". This wave vector is decomposed into a transverse component $k_{t}$ and a $z$-axis component $\gamma=\alpha+j \beta$, where $\alpha$ represents the attenuation constant and $\beta$ the propagation constant along $z$-axis. A wave propagation toward positive $\mathrm{z}$-axis is here represented using the convention $e^{-\gamma z}$.

To satisfy the boundary condition for the propagation, $\gamma$ should be identical in all layers. Knowing that the real part of $\varepsilon_{\text {skin }}$ is greater than the ones of $\varepsilon_{\text {air }}$ and $\varepsilon_{f a t}$, and since the muscle layer is considered infinite, propagating waves are mostly confined in the skin, as indicated by red arrows in Fig. 1, forming a stationary wave in the transverse direction. In the tissues surrounding the skin, the waves should exhibit an exponential transverse decay behavior. To determine the dispersion within the structure, the Transverse Resonance Method (TRM) is used [16]. For a propagation mode to exist, $k_{t}$ should satisfy the TRM condition: $Z_{\text {air }}+Z_{e q}=0$, where, $Z_{e q}$ represents the impedance exhibited by the tissues at the skin/air interface. The transverse impedance of a homogenous layer " $i$ " is given by $Z_{T M}^{i}=\frac{k_{t}}{\omega \varepsilon_{i}}$ for TM mode (magnetic field normal to the $x-z$ plane) and $Z_{T E}^{i}=\frac{\omega \mu_{i}}{k_{t}}$ for TE mode (electric field normal to the $x-z$ plane). Complex solutions $\gamma$ of these complex equations were found numerically in order to plot the dispersions diagram. To validate the code, obtained results have been compared with CST Microwave Studio in the case of lossless tissues with an eigenmode analysis and in the case of lossy tissues with the port mode solver. An agreement better than $0.1 \%$ has been observed on both the propagation constant value and the attenuation constant. Using these solutions and by expressing the boundary conditions at each interface between tissues, electric field $\boldsymbol{E}$ and magnetic field $\boldsymbol{H}$ can be determined. The Poynting vector is therefore calculated in order to illustrate the energy distribution of the modes in the next section.

\section{RESULTS}

\section{A. Dispersive Analysis}

Dispersion and attenuation for modes propagating up to $60 \mathrm{GHz}$ are calculated and results are shown in Fig. 2a for a $0.5 \mathrm{~mm}$-thick skin and a $13 \mathrm{~mm}$-thick fat layer. The first mode is a TE mode, starting propagating at about $8 \mathrm{GHz}$, which is confined within the skin layer. In addition, two other solutions are found which have a propagation constant very close, but smaller, to $k_{0}$ and to $k_{f a t}$, respectively. The first solution represents a fast wave with respect to the semi-infinite air medium while the second one represents a fast wave with respect to the semi-infinite fat medium. Both are proper solutions and represent the so-called Zenneck surface waves (ZW) at the air/skin and fat/skin interfaces respectively.
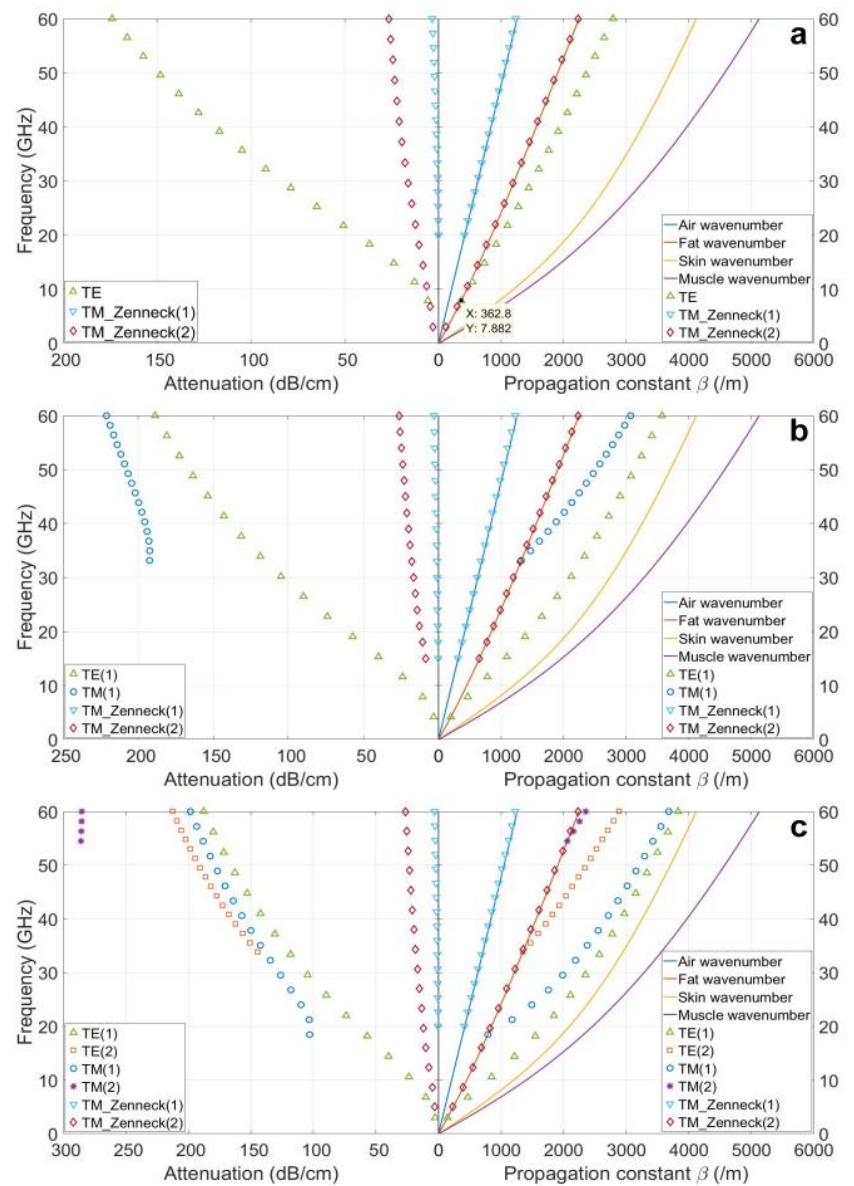

Fig. 2. Propagation and attenuation constants in the direction of propagation for three different skin thicknesses $(0.5 \mathrm{~mm}, 1 \mathrm{~mm}, 1.5 \mathrm{~mm}$ ) (fat thickness is $13 \mathrm{~mm}$ ) 
However, ZW cannot be excited separately from other surface-wave contribution when using a finite-size source [17]. Moreover, as shown later in section III-C, although these solutions contribute to describe the total field distribution, they are too loosely bounded to the interface to be useful for on-body communication.

Fig. $2 \mathrm{~b}$ and $2 \mathrm{c}$ show the same results when the skin thickness is increased to $1 \mathrm{~mm}$ and $1.5 \mathrm{~mm}$ respectively. The same $\mathrm{ZW}$ solutions are naturally found. As expected, the fundamental TE mode has a cut-off frequency that decreases when skin thickness increases. Moreover, as the frequency increases, higher order propagation modes appear successively. The cut-off frequencies are shown in Table. I. In the three studied models, the second TE mode is only found with the $1.5 \mathrm{~mm}$-thick skin model, and the first TM mode only with the $1 \mathrm{~mm}$-thick and $1.5 \mathrm{~mm}$-thick skin models.

TABLE I

CUT-OFF FREQUENCIES OF DiFFERENT MODES FOR THREE DIFFERENT MODELS

\begin{tabular}{|l|l|l|l|l|}
\hline Skin thickness & TE(1) & TM(1) & TE(2) & TM(2) \\
\hline $0.5 \mathrm{~mm}$ & $8 \mathrm{GHz}$ & - & - & - \\
\hline $1.0 \mathrm{~mm}$ & $4 \mathrm{GHz}$ & $33 \mathrm{GHz}$ & - & - \\
\hline $1.5 \mathrm{~mm}$ & $3 \mathrm{GHz}$ & $18 \mathrm{GHz}$ & $34 \mathrm{GHz}$ & $54 \mathrm{GHz}$ \\
\hline
\end{tabular}

The attenuation of each mode along the direction of propagation is given in terms of $\mathrm{dB} / \mathrm{cm}$ on the left-hand side of the figures. Only the fundamental TE mode exhibits reasonable attenuation for frequencies below $8 \mathrm{GHz}$, which could be suitable for on-body ranging transmission (not considering $\mathrm{ZW}$ ). As the frequency increases, its attenuation becomes significantly larger. Higher order propagating modes have always larger attenuations. Fig. 3 shows the attenuation with respect to frequency of the TE fundamental mode for six different skin thicknesses, ranging from 0.5 to $3 \mathrm{~mm}$. It is noted that, at a given frequency, thinnest skins exhibit the lowest attenuation.

Sensitivity of today's receiver could then allow communication in the range of few tens of centimeters.

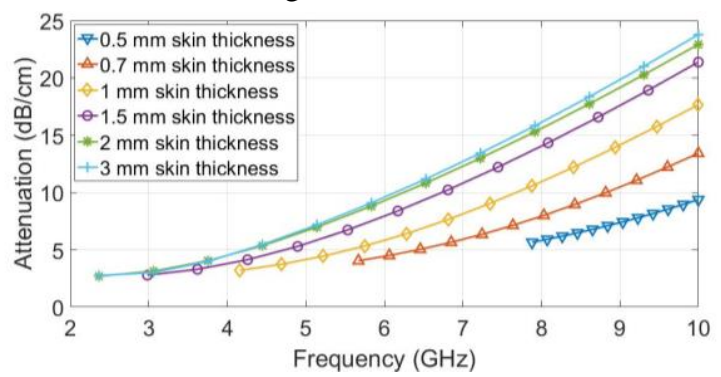

Fig. 3. Attenuation of the TE fundamental mode for different skin thickness models $(0.5-3 \mathrm{~mm}$ ) up to $10 \mathrm{GHz}$ (fat thickness is $13 \mathrm{~mm}$ )

\section{B. SW confinement}

All solutions but Zenneck ones have a propagation constant $\beta$ ranging between fat wavenumber $k_{f a t}$ and skin wavenumber $k_{\text {skin }}$, and represent consequently slow waves (SWs) confined within the skin. However, it is to be noted that since $\operatorname{Re}\left(\varepsilon_{\text {muscle }}\right)>\operatorname{Re}\left(\varepsilon_{\text {skin }}\right)$, a SW that propagates within the skin would also propagate within the muscle. However, the surface wave launcher is likely to be located at the skin-air interface. So if the transverse attenuation within the fat is large enough, the amount of the SW energy guided within the muscle layer shall be small. To quantify this assumption, the transverse attenuation within the fat layer is given in Fig. 4 for the fundamental TE mode. It can be observed that the attenuation is always greater than $5 \mathrm{~dB} / \mathrm{cm}$ in the $4-10 \mathrm{GHz}$ range of interest. Thus, depending on the fat thickness, the SW will not leak much in the muscle tissue.

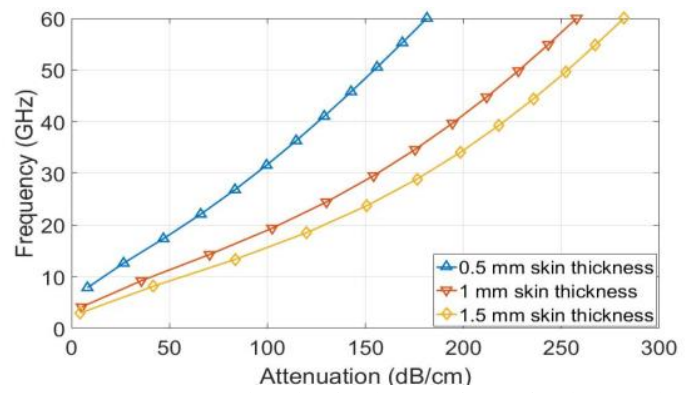

Fig. 4. Transverse attenuation in fat layer of TE fundamental mode for different skin thickness models $(0.5 \mathrm{~mm}, 1 \mathrm{~mm}, 1.5 \mathrm{~mm})$ up to $10 \mathrm{GHz}$ (fat thickness is $13 \mathrm{~mm}$ )

To investigate the confinement of the SW within the skin with respect to the field decay in the air, Fig. 5 shows the transverse attenuation constant in the air for the fundamental TE mode. In the frequency ranging below $10 \mathrm{GHz}$, the field decays away by about $15 \mathrm{~dB} / \mathrm{cm}$ and up to $70 \mathrm{~dB} / \mathrm{cm}$ as the frequency increases. Such an attenuation ensures that on-body SW propagation is confined enough in order to avoid any eavesdropper to intercept the communication. It is also observed that this attenuation is more than twice as high as the attenuation along the direction of propagation.

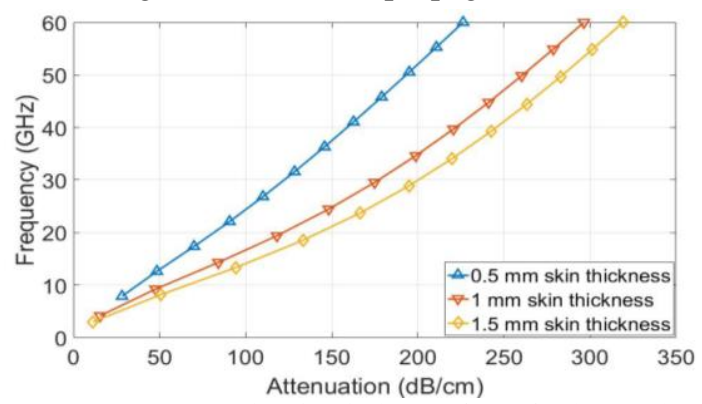

Fig. 5. Transverse attenuation in air layer of TE fundamental mode for different skin thickness models $(0.5 \mathrm{~mm}, 1 \mathrm{~mm}, 1.5 \mathrm{~mm})$ up to $10 \mathrm{GHz}$ (fat thickness is $13 \mathrm{~mm}$ )

\section{Field Distribution}

Fig. 6 exhibits the power distributions of the fundamental TE mode at $4 \mathrm{GHz}$ and $6 \mathrm{GHz}$ in the case of a $1 \mathrm{~mm}$-thick skin. It is clearly visible that operating at $6 \mathrm{GHz}$ exhibits better skin-confinement property, but the attenuation in the direction of propagation is also greater. TE mode at $4 \mathrm{GHz}$ has a $3.2 \mathrm{~dB} / \mathrm{cm}$ attenuation in the direction of propagation, $15.9 \mathrm{~dB} / \mathrm{cm}$ and $5.7 \mathrm{~dB} / \mathrm{cm}$ in the transverse direction in the air layer and fat layer respectively. As for $6 \mathrm{GHz}$, the attenuations
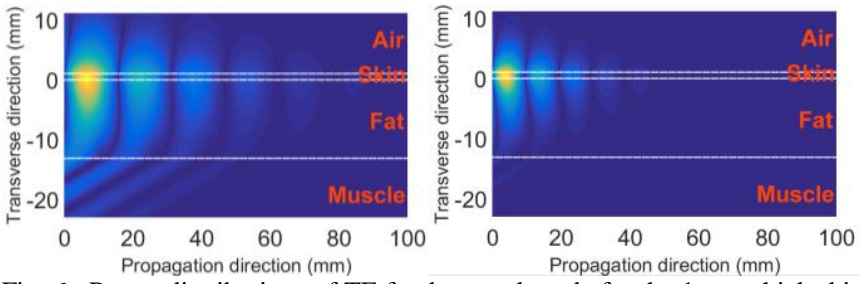

Fig. 6. Power distributions of TE fundamental mode for the $1 \mathrm{~mm}$-thick skin and $13 \mathrm{~mm}$-thick fat model at $4 \mathrm{GHz}$ (left) and $6 \mathrm{GHz}$ (right) 
in the direction of propagation, in the transverse direction in the air and fat layers are $5.8 \mathrm{~dB} / \mathrm{cm}, 26.1 \mathrm{~dB} / \mathrm{cm}$, and $15.0 \mathrm{~dB} / \mathrm{cm}$ respectively. The transmission frequency should then be chosen considering a tradeoff between the confinement and attenuation requirements, knowing that the confinement property is associated with the transferred data security and the attenuation in the direction of propagation involves the range and data rate of the wireless system.

To better illustrate the behavior of the two Zenneck surface wave poles, their power distribution is shown in Fig. 7 for the $1 \mathrm{~mm}$-thick skin model at $6 \mathrm{GHz}$. The first $\mathrm{ZW}$ propagating at the air/skin interface is loosely bounded and exhibits approximately no transverse attenuation in the air. The second $\mathrm{ZW}$ propagates at the skin/fat interface and has a low transverse attenuation in the fat layer $(0.04 \mathrm{~dB} / \mathrm{cm}$ in fat and $21.7 \mathrm{~dB} / \mathrm{cm}$ in air). Consequently, despite their low attenuation in the direction of propagation, these two modes do not exhibit any confinement properties. More importantly, it is commonly accepted that these types of modes cannot be excited independently [17].
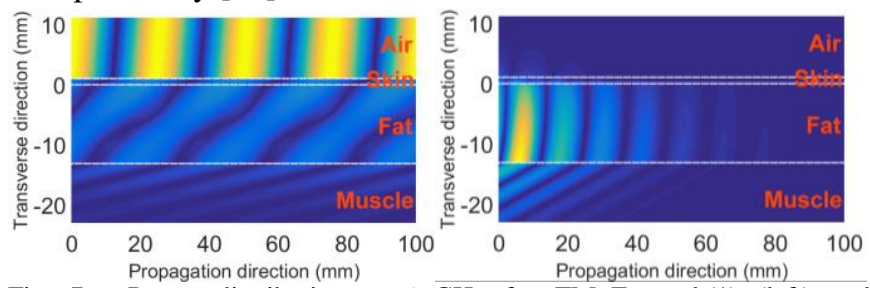

Fig. 7. Power distribution at $6 \overline{\mathrm{GHz}}$ for TM_Zenneck(1) (left) and TM_Zenneck(2) (right) of $1 \mathrm{~mm}$-thick skin and $13 \mathrm{~mm}$-thick fat model

\section{Fat Thickness Influence}

To study the influence of the muscle proximity to the skin on the fundamental TE mode, different thicknesses of fat ranging from $1 \mathrm{~mm}$ to $13 \mathrm{~mm}$ are studied, with the skin thickness fixed at $1 \mathrm{~mm}$. The propagation and attenuation constants are given as a function of fat thickness in Fig. 8, with a frequency ranging from $4.5 \mathrm{GHz}$ to $8 \mathrm{GHz}$. As shown previously, the propagation is less confined in skin at lower frequency, which makes it more vulnerable to the reflection from the fat/muscle interface. When the fat thickness decreases, larger attenuation and smaller $\beta$ are observed at a given frequency. The confined propagation is less sensitive to the change of fat thickness at higher frequency. Also, the cut-off frequency increases when the fat thickness decreases. No confined mode was found in the model with a fat thickness less than $10 \mathrm{~mm}$ at $4.5 \mathrm{GHz}, 5 \mathrm{~mm}$ for $5 \mathrm{GHz}$, and $3 \mathrm{~mm}$ for $6 \mathrm{GHz}$. The thickness of fat noticed in medical articles varies from $1 \mathrm{~mm}$ to $50 \mathrm{~mm}$ [15], so the operating frequency should be used with respect to the expected location

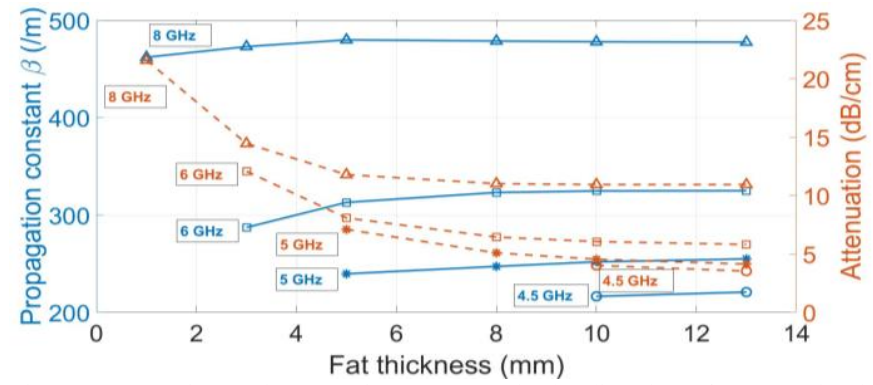

Fig. 8. Propagation and attenuation constants in the direction of propagation in function of fat thickness (skin thickness is $1 \mathrm{~mm}$ ) of sensors on the body, depending on the targeted application.

\section{CONCLUSION}

In this work, a dispersion analysis of the propagating modes within human tissues has been presented using transverse resonance method. The influence of the different tissue layers has been highlighted. It has been observed that operating in the 3-10 GHz frequency range could allow short range propagation within the human skin. This type of communication would naturally enable secure on-body communications to ensure data confidentiality in BANs.

The perspective of this work is to design on-body surface wave launchers in order to verify whether the identified fundamental TE mode can be efficiently excited.

\section{REFERENCES}

[1] "IEEE Standard for Local and metropolitan area networks - Part 15.6: Wireless Body Area Networks," IEEE Std 802156-2012, pp. 1-271, Feb. 2012.

[2] H. Liu et al., "Performance Assessment of IR-UWB Body Area Network (BAN) Based on IEEE 802.15.6 Standard," IEEE Antennas Wirel. Propag. Lett., vol. 15, pp. 1645-1648, 2016.

[3] L. Roelens, S. V. den Bulcke, W. Joseph, G. Vermeeren, and L. Martens, "Path loss model for wireless narrowband communication above flat phantom," Electron. Lett., vol. 42, no. 1, p. 10-11-, Jan. 2006.

[4] M. A. Callejón, D. Naranjo-Hernández, J. Reina-Tosina, and L. M. Roa, "A Comprehensive Study Into Intrabody Communication Measurements," IEEE Trans. Instrum. Meas., vol. 62, no. 9, pp. 24462455, Sep. 2013.

[5] A. Lea, P. Hui, J. Ollikainen, and R. G. Vaughan, "Propagation Between On-Body Antennas," IEEE Trans. Antennas Propag., vol. 57, no. 11, pp. 3619-3627, Nov. 2009.

[6] T. Alves, B. Poussot, and J. M. Laheurte, "Analytical Propagation Modeling of BAN Channels Based on the Creeping-Wave Theory," IEEE Trans. Antennas Propag., vol. 59, no. 4, pp. 1269-1274, Apr. 2011.

[7] L. Petrillo, T. Mavridis, J. Sarrazin, A. Benlarbi-Delaï, and P. D. Doncker, "Statistical On-Body Measurement Results at $60 \mathrm{GHz}$," IEEE Trans. Antennas Propag., vol. 63, no. 1, pp. 400-403, Jan. 2015.

[8] W. Thompson, K. Walker, R. Cepeda, M. A. Beach, and J. McGeehan, "Ultra wideband body area network channel measurement and analysis using textile antennas," in 2009 2nd IET Seminar on Antennas and Propagation for Body-Centric Wireless Communications, 2009, pp. 1-5.

[9] C. T. Ong, J. Y. Hee, T. S. P. See, and L. C. Ong, "Propagation studies of UWB transmission on human arm," in 2008 Asia-Pacific Microwave Conference, 2008, pp. 1-4.

[10] M. Grimm and D. Manteuffel, "On-Body Antenna Parameters," IEEE Trans. Antennas Propag., vol. 63, no. 12, pp. 5812-5821, Dec. 2015.

[11] "Dielectric Properties of Body Tissues [Online]. Available: http://niremf.ifac.cnr.it/tissprop/." .

[12] M. M. Black, "A Modified Radiographic Method for Measuring Skin Thickness*," Br. J. Dermatol., vol. 81, no. 9, pp. 661-666, Sep. 1969.

[13] H. Alexander and D. L. Miller, "Determining Skin Thickness with Pulsed Ultra Sound," J. Invest. Dermatol., vol. 72, no. 1, pp. 17-19, Jan. 1979.

[14] R. Marks, P. J. Dykes, and E. Roberts, "The measurement of corticosteroid induced dermal atrophy by a radiological method," Arch. Dermatol. Res., vol. 253, no. 2, pp. 93-96.

[15] H. Zahouani, C. Pailler-Mattei, B. Sohm, R. Vargiolu, V. Cenizo, and R. Debret, "Characterization of the mechanical properties of a dermal equivalent compared with human skin in vivo by indentation and static friction tests," Skin Res. Technol. Off. J. Int. Soc. Bioeng. Skin ISBS Int. Soc. Digit. Imaging Skin ISDIS Int. Soc. Skin Imaging ISSI, vol. 15, no. 1, pp. 68-76, Feb. 2009.

[16] D. M. Pozar, Microwave Engineering, 4th Edition. Hoboken, NJ: John Wiley \& Sons, 2011.

[17] K. A. Michalski and J. R. Mosig, "The Sommerfeld Halfspace Problem Redux: Alternative Field Representations, Role of Zenneck and Surface Plasmon Waves," IEEE Trans. Antennas Propag., vol. 63, no. 12, pp. 5777-5790, Dec. 2015. 